\title{
A Comparison of the Efficacy and Safety of Non-Steroidal Anti-Inflammatory Drugs versus Acetaminophen in Symptom Relief for the Common Cold: A Meta-Analysis of Randomized Controlled Trial Studies

\section{Il-Kwon Choi, Hyun-Keun Lee, Young-Jung Ji, In-Hong Hwang, Soo Young Kim*}

Department of Family Medicine, Kangdong Sacred Heart Hospital, Hallym University College of Medicine, Seoul, Korea

Background: Non-steroidal anti-inflammatory drugs (NSAIDs) and acetaminophen are widely used for common cold symptom relief. The objective of this study was to evaluate and compare the efficacy and safety of acetaminophen and NSAIDs in common cold symptom relief using meta-analysis of randomized controlled trial.

Methods: We searched MEDLINE (PubMed), Cochrane, EMBASE, CINAHL, KMbase, KoreaMed, National Assembly Library, and Riss4u for studies released through June 2012. Two authors independently extracted the data. To assess the risk of bias, the Cochrane Collaborations risk of bias tool was used. The Review Manager ver. 5.1 (RevMan) was used for statistics.

Results: We identified 5 studies. The relative benefit for participants with pain relief was 1.00 (95\% confidence interval [CI], 0.96 to 1.05 ) and $\mathrm{I}^{2}=0 \%$. The existence of the heterogeneity between studies was not important in this study, thus subgroup analysis was not implemented. The relative benefit for participants with rhinorrhea was 1.02 (95\% CI, 0.77 to 1.35 ) and $\mathrm{I}^{2}=0 \%$, which also indicates the existence of heterogeneity was not important. The relative risk of adverse events was 1.14 (95\% CI, 0.93 to 1.40$), \mathrm{I}^{2}=0 \%$. There was no apparent asymmetry in the funnel plot.

Conclusion: There was no difference between NSAIDs and acetaminophen in common cold symptom relief.

Keywords: Common Cold; Non-Steroidal Anti-Inflammatory Agents; Acetaminophen; Meta-Analysis

Received: August 31, 2012, Accepted: May 8, 2013

*Corresponding Author: Soo Young Kim

Tel: +82-2-2224-2406, Fax: +82-2-2224-2409

E-mail: hallymfm@gmail.com

Korean Journal of Family Medicine

Copyright (C) 2013 The Korean Academy of Family Medicine

(a) This is an open-access article distributed under the terms of the Creative Commons Attribution Non-Commercial License (http://creativecommons.org/licenses/by-nc/3.0) which permits unrestricted noncommercial use, distribution, and reproduction in any medium, provided the original work is properly cited.

\section{INTRODUCTION}

The common cold is an acute respiratory tract infection and is a common and widespread disease in all ages. Typically, it shows mild symptoms but if it persists over a week, out-patient treatment may be needed. ${ }^{1)}$ The common cold presents acute symptoms such as cough, runny nose, sneezing, sore throat, and hoarseness, accompanied by fever, chill, muscle pain, and headache. Among them, nasal symptoms are the most common. ${ }^{2)}$

Although in most cases, a virus is the cause of the common 
cold, anti-viral treatment is not appropriate in Korea, as antiviral medicine administration for the common cold is not approved by the Korea Food and Drug Administration and is not available in Korea. ${ }^{3-5)}$ As the causal treatment of the common cold is difficult, the purpose of common cold treatment is relief of various symptoms. As symptoms are resolved in 7 to 10 days spontaneously, allopathy is usually adopted. ${ }^{6)}$ Representative allopathic treatment includes prescription of non-steroidal antiinflammatory drugs (NSAIDs) and acetaminophen to resolve chills, muscle pain, headache, and fever. ${ }^{1)}$

A meta-analysis in 2009 reported that NSAIDs did not give much relief for other symptoms, although they were helpful for pains from the common cold, ${ }^{5)}$ and there were reports that acetaminophen was effective against upper airway infections, especially symptoms such as sore throat, ${ }^{7)}$ headache, ${ }^{8)}$ and fever. ${ }^{9)}$

On the other hand, according to many studies, it is common for NSAIDs to have adverse effects such as peptic ulcer and gastrointestinal disorders, and a recent meta-analysis reported that they increase the risk of myocardial infarction and cardiac insufficiency, and adverse effects are dose proportional. ${ }^{10-12)} \mathrm{On}$ the other hand, not many adverse effects were reported with acetaminophen at normal dosage, and it was reported to have relatively less gastrointestinal adverse effects than NSAIDs. ${ }^{13)}$ Until now, the choice between two medications to mitigate the symptoms of the common cold has been made according to the characteristics and preference of the doctor or the patient, and there has not been a meta-analysis of randomized controlled studies that comprehensively compared the effects of the two medications. Thus, the purpose of this study was to compare the effects and safety of NSAIDs and acetaminophen in the relief of symptoms of the common cold through a meta-analysis of randomized controlled trials.

\section{METHODS}

In order to compare the efficacy and safety of NSAIDs and acetaminophen on common cold patients, a meta-analysis was performed on randomized controlled trials.

\section{Inclusion Criteria}

Published randomized controlled trials comparing the effects of oral NSAIDs versus acetaminophen on common cold symptom relief were included. The common cold was defined by symptoms of runny nose, nasal congestion and sneezing, regardless of symptoms of headache and coughs. Studies on patients diagnosed as other than common cold, such as acute sinusitis, exudative tonsilitis, acute laryngitis, tracheitis, acute laryngotracheobronchitis, epiglottitis, tympanitis lower airway infectious diseases (pneumonia, bronchitis and so on), chronic respiratory disease, or allergic rhinitis were excluded. Studies using oral NSAIDs and oral acetaminophen for the treatment of the common cold were included regardless of dosage form or amount, but single-dose studies were excluded. Studies reporting overall evaluation of relief of common cold symptoms, analgesic effects, and nonanalgesic effects such as runny nose or cough were included.

\section{Searches}

The final search was made in June, 2012. A professional librarian made the search. The key words were selected through discussion between authors and the librarian. There was no restriction on the language of published articles.

Searches were made on MEDLINE (PubMed), EMBASE, the Cochrane Central Register of Controlled Trials (CENTRAL), and CINAHL for studies released through June 2012. For domestic studies, KoreaMed, KMbase, National Assembly Library and Riss $4 u$ were searched. Keywords were selected to include common cold-related, NSAIDs-related, acetaminophenrelated, and randomized controlled trial-related words. Detailed keywords are shown in Appendix 1. Additional searches were made for the references of the included articles.

\section{Studies Selection}

Two independent authors selected studies satisfying the inclusion criteria from the search results, and disagreement was settled by discussion and consensus between two authors. When agreement could not be made, final selection was made with the mediation of a third author.

\section{Assessment of Risk of Bias in Included Studies}

For the methodological quality evaluation of the included studies, two independent authors made assessment using the Cochrane Collaboration's tool for assessing risk of bias. ${ }^{14)}$ Each 
item was classified as low risk, high risk, or unclear; low risk for low risk of bias, high risk for high risk of bias, and unclear for difficult to decide. We resolved disagreements by discussion.

\section{Data Extraction}

Two independent authors independently carried out data extraction using standard data extraction forms. When authors did not agree, two authors tried to find consensus through discussion.

\section{Statistics Analysis}

Relative benefit $(R B)$ or relative risk $(R R)$ was proposed with $95 \%$ confidence interval (CI). To analyze the study results, Review Manager ver. 5.0 (RevMan; Cochrane Collaboration, Oxford, UK), ${ }^{15)}$ a statistics program was used. The results were summarized in a table through forest plot, and funnel plot was applied to check for publication bias.

To identify heterogeneity, the $\mathrm{I}^{2}$ test was used. $\mathrm{I}^{2}$ statistics are the form of statistics quantifying inconsistency. $\mathrm{I}^{2}$ ranges from $0 \%$ to $100 \%$. Here, values between $0 \%$ and $40 \%$ can be interpreted as unimportant heterogeneity, up to $60 \%$ as moderate heterogeneity, and over $60 \%$ as considerable heterogeneity. ${ }^{16)}$ When heterogeneity did not exist, it was analyzed as a fixedeffect model. When heterogeneity existed, the group was divided into subgroups with similar characteristics to investigate reasons for heterogeneity. If the heterogeneity could not be resolved, a random effect model was applied. When heterogeneity was not significant, subgroup analysis was not performed.

\section{RESULTS}

\section{Description of Studies}

Sixty-one articles were retrieved through an electronic search, 35 articles in MEDLINE (PubMed), 4 articles in Cochrane, 15 articles in EMBASE, and 7 articles in CINAHL. There were no domestic articles. Fifty-eight abstracts excluding redundant studies were screened. Of these, 5 studies met the inclusion criteria: Moore et al., ${ }^{17)}$ Ulukol et al., ${ }^{18)}$ Ugazio et al., ${ }^{19)}$ Graham et al., ${ }^{20)}$ and Phadke et al. ${ }^{21)}$ (Figure 1).

A total of 3,074 participants were included. The NSAIDs group included 2,033 participants and the acetaminophen group included 1,041. In all studies, the patients showed clinical symptoms of the common cold, and the gender ratio was similar. Symptom relief effects and adverse effects of medications were reported from the third day to the 14th day of administration (Table 1).

\section{Risk of Bias in Included Studies}

When assessing the risk of bias using Cochrane Collaboration's tool for assessing risk of bias, ${ }^{14)}$ it was found that two studies performed adequate sequence generation. Only one study showed a low risk of bias in allocation concealment, two studies uncertain, and the remaining two studies a high risk of bias. Whether blinding was appropriate in two studies, two other studies did not perform blinding properly. Regarding

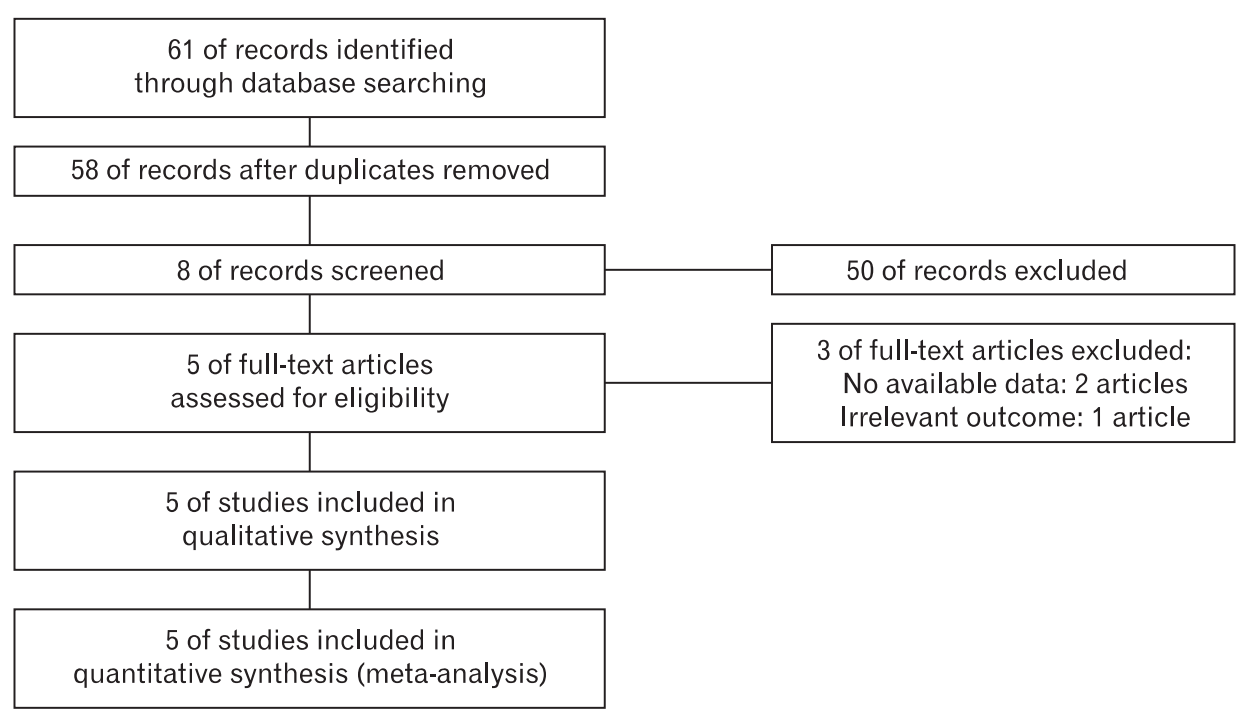

Figure 1. Flow sheet of study selection. 


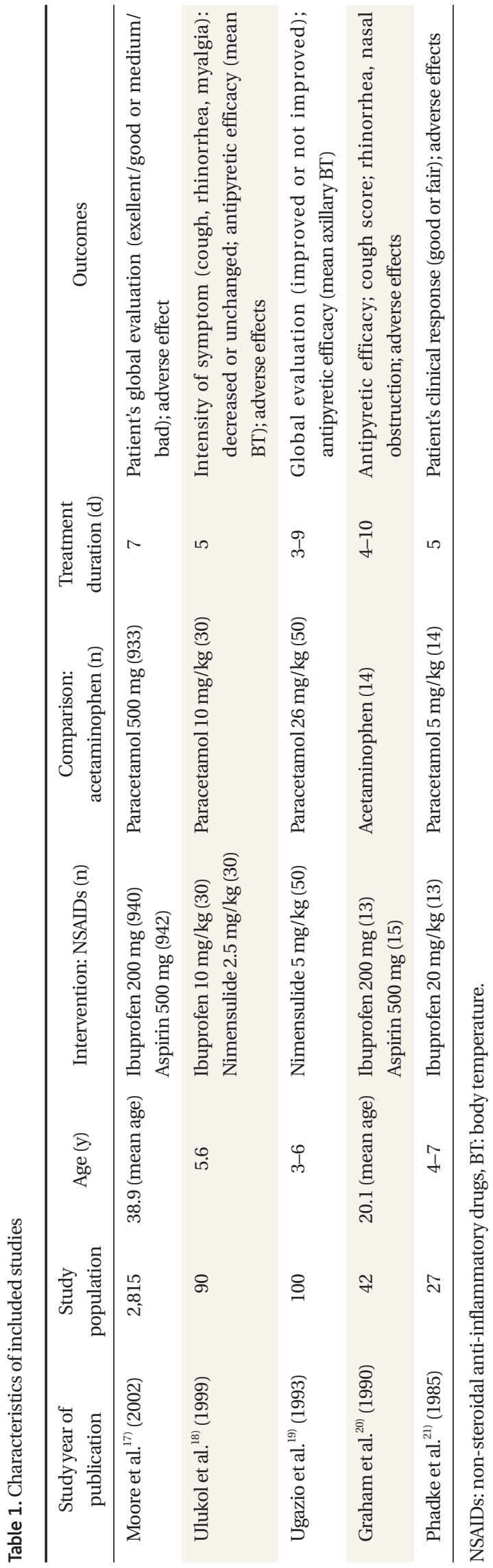

incomplete outcome data reporting, all the studies except 1 study were uncertain, because they did not report enough to be judged as satisfying the criteria. Selective reporting could not be determined as all of the studies did not report the protocol. For other biases, two studies were evaluated as unclear because they did not provide accurate data for each group (Figure2).

\section{Effects of Intervention}

\section{1) Analgesic effects}

Three trials reported the analgesic effect of NSAIDs and acetaminophen in the common cold. ${ }^{17,19,21)} \mathrm{RB}$ of the NSAIDs group versus the acetaminophen group in analgesic effect in the common cold was 1.00 (95\% CI, 0.96 to 1.05 ), and $\mathrm{I}^{2}$ was $0 \%$ (Figure 3). As there was no heterogeneity among studies, subgroup analysis was not performed.

2) Non-analgesic effects (decrease of rhinorrhea)

Two studies ${ }^{18,20)}$ measured outcomes other than the analgesic effect. All reported rhinorrhea decrease effect of acetaminophen

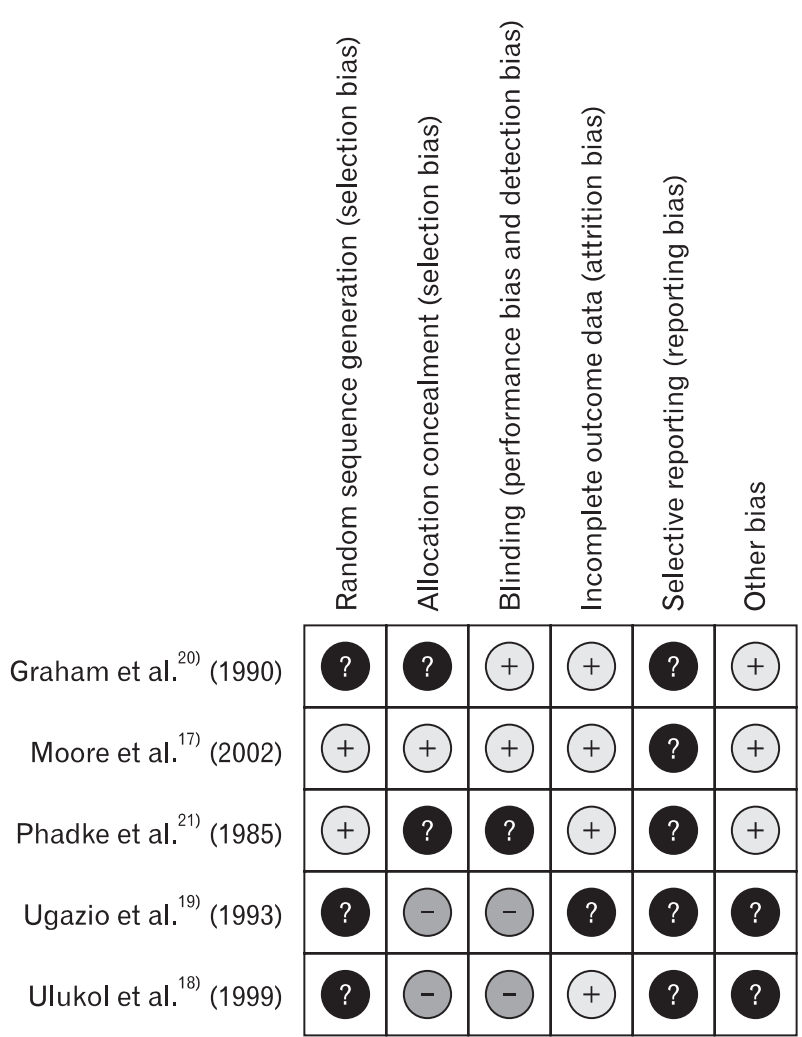

Figure 2. Risk of bias summary. 


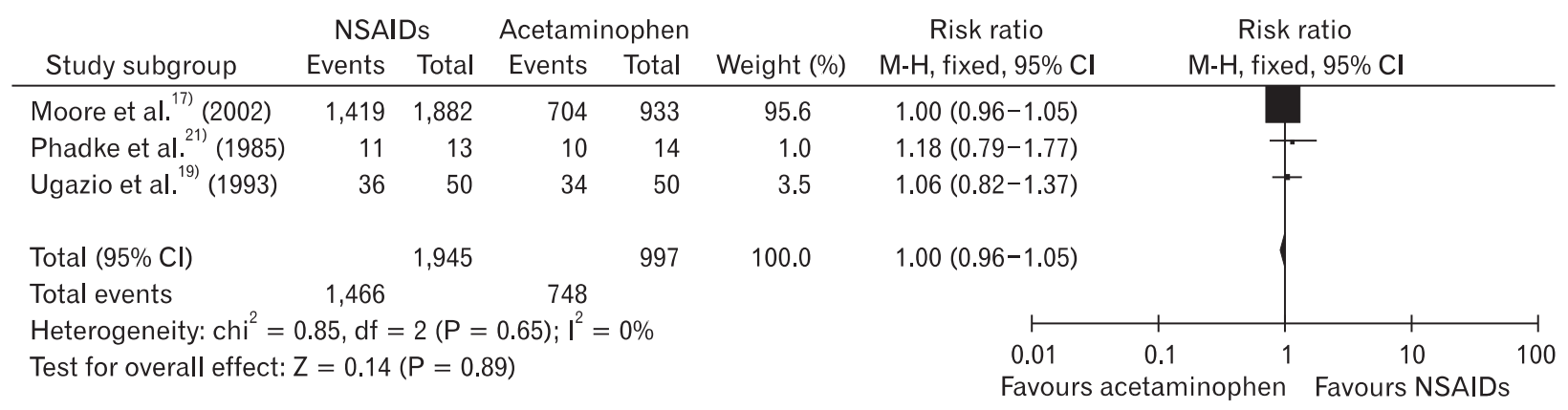

Figure 3. Non-steroidal anti-inflammatory drugs (NSAIDs) versus acetaminophen: analgesic effects. Participants with pain relief. CI: confidence interval.

\begin{tabular}{|c|c|c|c|c|c|c|c|c|}
\hline \multirow[b]{2}{*}{ Study subgroup } & \multicolumn{2}{|c|}{ NSAIDs } & \multicolumn{2}{|c|}{ Acetaminophen } & \multirow[b]{2}{*}{ Weight (\%) } & \multirow{2}{*}{$\begin{array}{c}\text { Risk ratio } \\
\mathrm{M}-\mathrm{H} \text {, fixed, } 95 \% \mathrm{Cl}\end{array}$} & \multirow{2}{*}{$\begin{array}{c}\text { Risk ratio } \\
\mathrm{M}-\mathrm{H} \text {, fixed, } 95 \% \mathrm{Cl}\end{array}$} & \\
\hline & Events & Total & Events & Total & & & & \\
\hline Graham et al. $^{20)}(1990)$ & 20 & 28 & 11 & 14 & 40.7 & $0.91(0.63-1.30)$ & & \\
\hline Ulukol et al. $^{18)}(1999)$ & 35 & 60 & 16 & 30 & 59.3 & $1.09(0.74-1.63)$ & & \\
\hline Total $(95 \% \mathrm{Cl})$ & & 88 & & 44 & 100.0 & $1.02(0.77-1.35)$ & & \\
\hline Total events & 55 & & 27 & & & & & \\
\hline \multirow{2}{*}{\multicolumn{5}{|c|}{ Heterogeneity: $\mathrm{chi}^{2}=0.51, \mathrm{df}=1(\mathrm{P}=0.48) ; \mathrm{I}^{2}=0 \%$}} & & & & \\
\hline & & & & & Test for overall effect: $Z=0.13(P=0.90)$ & 0.01 & 0.1 & 100 \\
\hline
\end{tabular}

Figure 4. Non-steroidal anti-inflammatory drugs (NSAIDs) vs. acetaminophen: nonanalgesic effects. Participants with rhinorrhea decreased. CI: confidence interval.

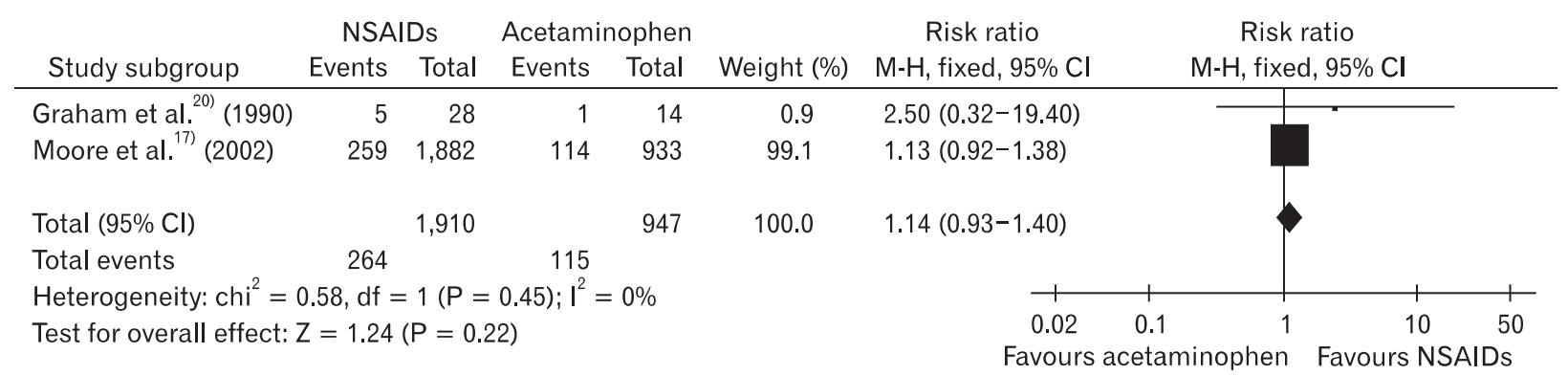

Figure 5. Non-steroidal anti-inflammatory drugs (NSAIDs) versus acetaminophen: adverse effect. Overall adverse effects. CI: confidence interval.

and NSAIDs. Among patients who experienced relief of symptoms other than pains, the RB of the NSAIDs group of patients who experienced rhinorrhea symptom relief to the acetaminophen group was 1.02 (95\% CI, 0.77 to 1.35 ), and $\mathrm{I}^{2}=0 \%$, which means there was no heterogeneity (Figure 4).

\section{3) Adverse effects}

Adverse effects were reported in 4 studies in total, but in two studies there were no patients who experienced adverse effects. Therefore, two studies ${ }^{17,20)}$ reported adverse effects. Most of adverse effects were trivial and the most frequent adverse effect was digestive system dysfunction. One study reported rectal hemorrhage in the NSAIDs group and hematemesis in the acetaminophen group. There was no statistically significant difference in adverse effects between the two medications but the NSAIDs group tended to experience more adverse effects. This is consistent with previous studies reporting relatively higher adverse effect cases in NSAIDs. RR of patients experiencing adverse effects was 1.14 (95\% CI, 0.93 to 1.40 ), and $\mathrm{I}^{2}=0 \%$ (Figure 5). 


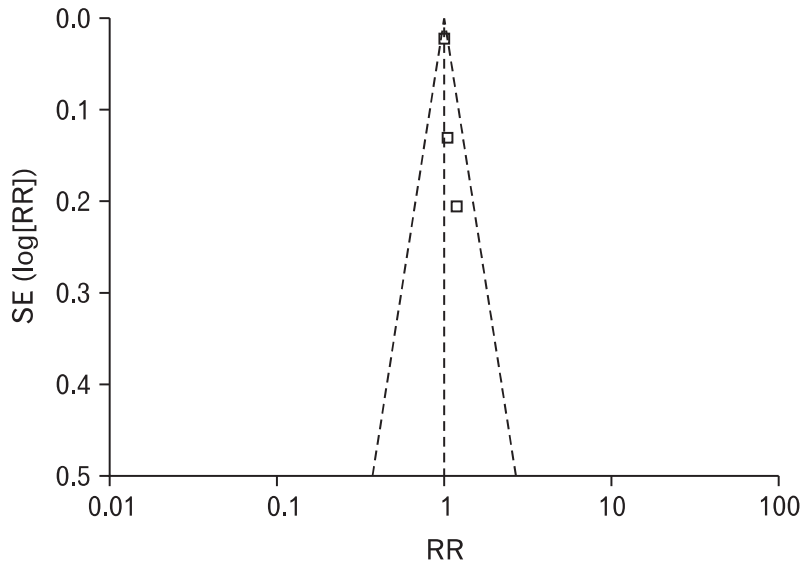

Figure 6. Funnel plot of included studies. SE: standard error, RR: relative risk.

\section{Publication Bias}

Obvious asymmetry was not observed in the funnel plot (Figure 6).

\section{DISCUSSION}

According to meta-analysis of five studies, there was no statistically significant difference in common cold symptom relief between acetaminophen and NSAIDs. As there was no heterogeneity, subgroup analysis was not made.

When the methodological quality of the included studies was evaluated using the Cochrane Collaboration's tool for assessing risk of bias, ${ }^{14)}$ the overall quality of studies was mixed, largely due to missing information regarding randomization procedures and selective reporting. We assessed one study ${ }^{17)}$ as being of high quality. Our outcomes were mainly subjective and blinding of participants may be critical. But the risk of bias regarding blinding was mixed.

In the literature review, the number of clinical studies regarding the comparison of interventions was not sufficient. Out of 3,074 patients included in this meta-analysis, 2,815 subjects were the participants of a study, ${ }^{17)}$ while the other 4 studies were relatively smaller in size. Among them, three studies ${ }^{18,19,21)}$ were on children and the other two studies ${ }^{17,20)}$ on adults. Accordingly the kinds and dose of medications used in the studies were various. No studies used an objective evaluation tool for outcome measure. Most of the studies judged mitigation effects according to subjective symptom reports of the patients. Although relief effects of individual symptoms varied by studies, overall judgment was made to determine the improvement. Accordingly, an article including more patients and using objective outcome measurement tools may be necessary in order to discover if the study results would be different according to subject group, intervention method or intervention results.

Although the two studies included in this meta-analysis judged that NSAIDs were more effective than acetaminophen in pain control, according to meta-analysis results there was no statistically significant difference in effects on common cold symptoms or adverse effects. Based on previous studies, high does NSAIDs could be relatively more effective for pain control. ${ }^{22)}$ However, more adverse effects on the gastrointestinal system were reported in NSAIDs. Therefore, the most appropriate medication should be selected considering that adverse effects of NSAIDs are proportional to dosage and there were less adverse effects from acetaminophen if they were used at the proper dose.

Therefore, when using NSAIDs rather than acetaminophen, care should be taken considering underlying conditions of the patient such as digestive troubles and cardiovascular disease. A large scale cohort randomized controlled clinical experiment is needed to identify the relationship between adverse effects and dosage, an important foundation for comparative selection between two medications.

\section{CONFLICT OF INTEREST}

No potential conflict of interest relevant to this article was reported.

\section{REFERENCES}

1. Heikkinen T, Jarvinen A. The common cold. Lancet 2003; 361:51-9.

2. Taverner D, Latte J. Nasal decongestants for the common cold. Cochrane Database Syst Rev 2007; (1):CD001953.

3. Treanor JJ, Hayden FG. Viral infections. In: Murray JF, Nadel JA, Mason RJ, Broaddus VC, editors. Textbook of respiratory medicine. 4th ed. Philadelphia: Saunders; 2005. p. 870-2. 
4. Desmond RA, Accortt NA, Talley L, Villano SA, Soong SJ, Whitley RJ. Enteroviral meningitis: natural history and outcome of pleconaril therapy. Antimicrob Agents Chemother 2006;50:2409-14.

5. Kim SY, Chang YJ, Cho HM, Hwang YW, Moon YS. Nonsteroidal anti-inflammatory drugs for the common cold. Cochrane Database Syst Rev 2009; (3):CD006362.

6. Tan T, Little P, Stokes T; Guideline Development Group. Antibiotic prescribing for self limiting respiratory tract infections in primary care: summary of NICE guidance. BMJ 2008;337:a437.

7. Schachtel BP, Fillingim JM, Thoden WR, Lane AC, Baybutt RI. Sore throat pain in the evaluation of mild analgesics. Clin Pharmacol Ther 1988;44:704-11.

8. Migliardi JR, Armellino JJ, Friedman M, Gillings DB, Beaver WT. Caffeine as an analgesic adjuvant in tension headache. Clin Pharmacol Ther 1994;56:576-86.

9. Bachert C, Chuchalin AG, Eisebitt R, Netayzhenko VZ, Voelker M. Aspirin compared with acetaminophen in the treatment of fever and other symptoms of upper respiratory tract infection in adults: a multicenter, randomized, double-blind, doubledummy, placebo-controlled, parallel-group, single-dose, 6-hour dose-ranging study. Clin Ther 2005;27:993-1003.

10. Juni P, Nartey L, Reichenbach S, Sterchi R, Dieppe PA, Egger M. Risk of cardiovascular events and rofecoxib: cumulative meta-analysis. Lancet 2004;364:2021-9.

11. Aldington S, Shirtcliffe P, Weatherall M, Beasley R. Increased risk of cardiovascular events with parecoxib/valdecoxib: a systematic review and meta-analysis. N Z Med J 2005;118: U1755.

12. Aw TJ, Haas SJ, Liew D, Krum H. Meta-analysis of cyclooxygenase-2 inhibitors and their effects on blood pressure. Arch Intern Med 2005; 165:490-6.

13. Lanas A, McCarthy D, Voelker M, Brueckner A, Senn S, Baron JA. Short-term acetylsalicylic acid (aspirin) use for pain, fever, or colds: gastrointestinal adverse effects: a metaanalysis of randomized clinical trials. Drugs RD 2011;11:27788.
14. Higgins JP, Green S. Assessing risk of bias in included studies. In: Higgins JP, Green S, editors. Cochrane handbook for systematic reviews of interventions version 5.1.0 [Internet]. Chichester: Wiley-Blackwell; 2011 [cited 2012 Aug 30]. Available from: http://www.cochrane-handbook.org.

15. Cochrane Collaboration. Review manager (RevMan) [computer program]. Version 5.1. Copenhagen: The Nordic Cochrane Centre; 2011.

16. Higgins JP, Altman DG. Analysing data and undertaking metaanalysis. In: Higgins JP, Green S, editors. Cochrane handbook for systematic reviews of interventions version 5.1.0 [Internet]. Chichester: Wiley-Blackwell; 2011 [cited 2012 Aug 30]. Available from: http://www.cochrane-handbook.org.

17. Moore N, Le Parc JM, van Ganse E, Wall R, Schneid H, Cairns R. Tolerability of ibuprofen, aspirin and paracetamol for the treatment of cold and flu symptoms and sore throat pain. Int J Clin Pract 2002;56:732-4.

18. Ulukol B, Koksal Y, Cin S. Assessment of the efficacy and safety of paracetamol, ibuprofen and nimesulide in children with upper respiratory tract infections. Eur J Clin Pharmacol 1999;55:615-8.

19. Ugazio AG, Guarnaccia S, Berardi M, Renzetti I. Clinical and pharmacokinetic study of nimesulide in children. Drugs 1993;46 Suppl 1:215-8.

20. Graham NM, Burrell CJ, Douglas RM, Debelle P, Davies L. Adverse effects of aspirin, acetaminophen, and ibuprofen on immune function, viral shedding, and clinical status in rhinovirus-infected volunteers. J Infect Dis 1990;162:127782.

21. Phadke MA, Paranjape PV, Joshi AS. Ibuprofen in children with infective disorders: antipyretic efficacy. Br J Clin Pract 1985;39:437-40.

22. Yoon YJ, Kim JH, Kim SY, Hwang IH, Kim MR. A comparison of efficacy and safety of non-steroidal anti-inflammatory drugs versus acetaminophen in the treatment of episodic tensiontype headache: a meta-analysis of randomized placebocontrolled trial studies. Korean J Fam Med 2012;33:262-71. 
Appendix 1. MEDLINE (PubMed), EMBASE search strategy and search strategy for domestic literatures

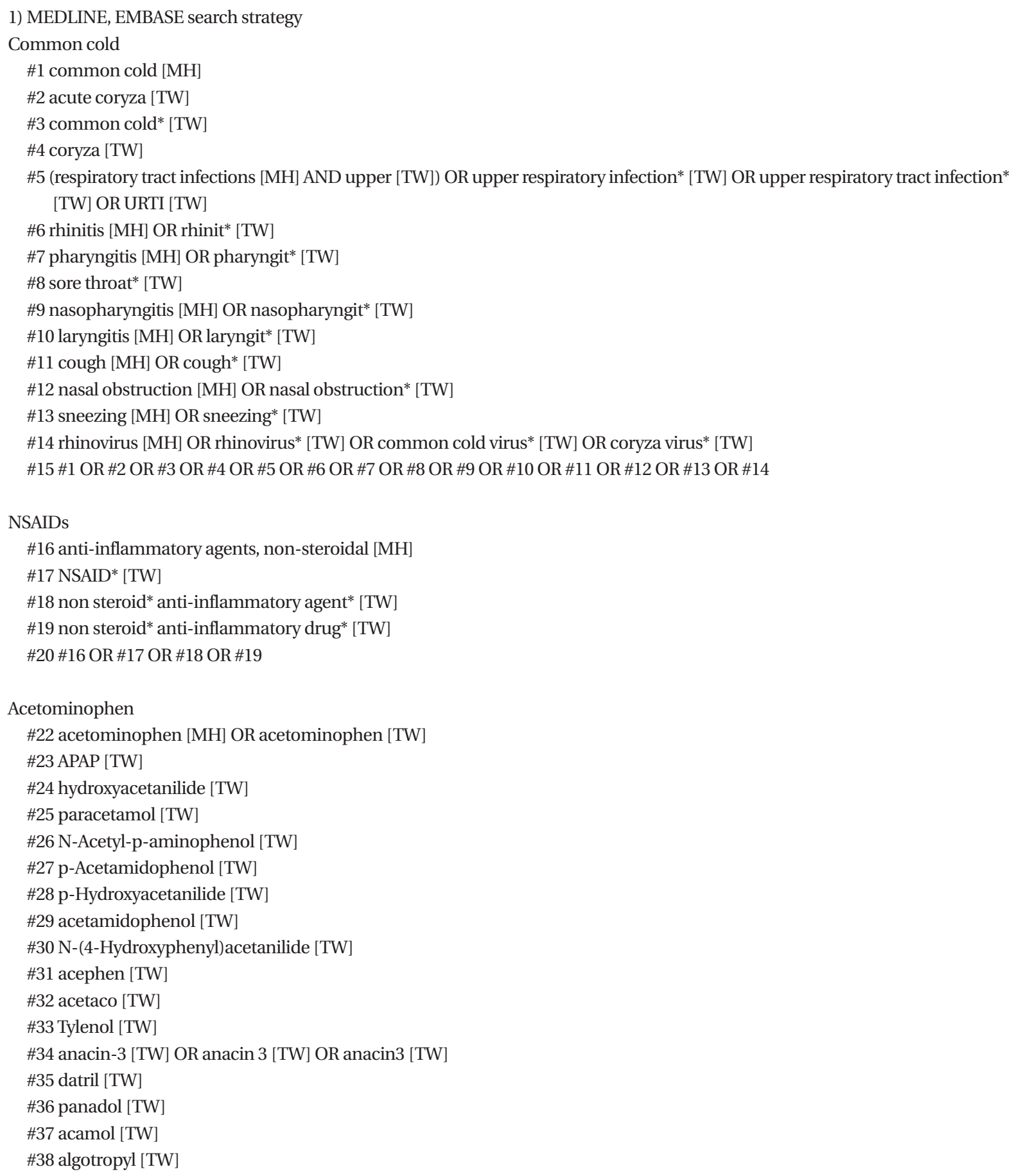


Appendix 1. Continued

2) Search strategy for domestic literatures

KMbase

\#1. 감기

\#2. NSAIDs

\#3. acetaminophen

\#4. \#1 1 AND \#2 AND \#3

\section{KoreaMed}

"common cold" [ALL] "acetaminophen" [ALL] / RCT [PT]

"common cold" [ALL] "NSAIDs" [ALL] / RCT [PT]

국회도서관, Riss $4 \mathrm{u}$

감기 AND NSAIDs AND acetaminophen 